\title{
Life strategies of selected algae species along the habitat gradient on peat bogs
}

\author{
Urszula Jacuńska
}

Department of Plant Ecology and Nature Conservation, Institute of Environment Protection, Nicolaus Copernicus University, Gagarina 9, 87-100 Toruń, Poland, e-mail: ujakub@biol.uni.torun.pl

\begin{abstract}
Autecological studies on some selected algae species were conducted in 1998 and 1999 on transition mires and raised bogs situated in the water and peat-bog nature reserve „Dury” in the Wdecki Landscape Park. Apart from forms co-dominating at all studied sites, such as Penium silvae-nigrae f. parallelum or Anisonema ovale, also forms that occurred occasionally were selected for autecological studies, such as e.g. Tetmemorus brebissonii var. brebissonii occurring in summer, or Chroococcus turgidus connected with sites situated near the water table. From the literature data it appears that the selected algae species, perhaps with exception of Petalomonas sphagnophila, are cosmopolitan organisms, characteristic of oligotrophic raised bogs. Species such as Anisonema ovale and Penium silvae-nigrae, despite different nutrition methods and different life ,strategies”, have a similarly wide range of tolerance to changeable habitat parameters. They are eurytopic species, which can find their developmental optimum in all habitats. Chroococcus turgidus and Tetmemorus brebissonii are autotrophic species, which display certain characteristics corresponding to the $\mathrm{K}$ - strategy of development. They are stenotopic species connected with a highly hydrated habitat of low electrolytic conductivity and low concentration of biogenic substances.

From the data presented in this paper, it appears that there exists a certain ecological group of algae adapted to life in raised bogs. It is a group, which finds its developmental optimum just on these habitats and which indeed deserves the term "sphagnum algae".
\end{abstract}

Key words: autecology Penium silvae-nigrae f. parallelum, Anisonema ovale, Chroococcus turgidus, Tetmemorus brebissonii and Petalomonas sphagnophila, peat bogs, Tuchola Forest, Poland.

\section{Introduction}

Autecological studies deal with reactions of organisms to factors of the broadly understood environment. On the one hand, the most important factors that definitely determine life functions of organisms, due to which they growth and preserve their biological structure for a sufficient time to reproduce, are resources of energy and matter available in their environment, whereas on the other hand - physical and chemical conditions of the substratum providing the indispensible elements of the organic structure. Due to the heterogeneity of the environment, such as a peat bog, the place occupied by a specimen can be a key determinant of its adaptation (Pianka 1981). As a consequence of the aforementioned situation, the population develops its own, unique response in the form of the so-called life strategy ensuring the survival in the environment, which is subject to continuous changes.

In the 1970 s, the dominant tendency in the research on the strategies of organisms was the division of the whole groups of characteristics and habitats, with which they are connected, into two contrary categories denoted as ,r" and „K” (MacArthur 1962). None of the species can be explicitly categorize into the strategy ,r” or „K”, each species takes a transitional position between these extreme cases. It is necessary to assume a certain continuum of the selection process $\mathrm{r} \rightarrow \mathrm{K}$, while the position of specific organisms in this continuum is variable and depends on the environment 
and the time (Lampert \& Sommer 1996). The results of the research performed on higher plants confirm the theory of a smooth transition of populations from one life strategy to another one - the most favourable at a given time and in specific habitat conditions (MacNaughton 1975; Law et al. 1977).

Margalef (1978), Kilham and Kilham (1980), as well as Smayda and Reynolds (2003), and Reynolds (2006), employed the notion of continuum of the reproduction strategy, from $r$ to $\mathrm{K}$, with reference to phytoplankton. Organisms subject to the strategy of the r-type live in the unstable environment and occur for a short time, e.g. in spring and early summer during the period of rapid physical and chemical transformations of the environment. Usually these are small species of a very short reproduction cycle, but also of a short life time. Species of the K strategy are much bigger; they grow more slowly and demonstrate the tendency to strong competition (Pianka 1. c., Sommer 1988; Turpin 1988). They dominate during the period of summer stagnation.

Grime (1979) quoted three types of adaptation strategies for terrestrial plants in relation to stress factors and factors disturbing the equilibrium. In 1988, these types were modified by Reynolds and applied for phytoplankton. According to this author, individual species of algae can be classified within one of the groups listed below. The first one consists of species with the „r strategy”, which grow quickly before other species in the environment saturated with sunlight and nutrients (according to Grime, they are competitors „C"). The second group comprises species of the „K strategy”, growing in the environment with significant deficiency of essential nutrients (according to Grime - species tolerant of stress „S”). The third group consists of species of the „strategy type w”, tolerant of continuous or frequent migration along the light gradient (according to Grime, these are species tolerant of disturbance, ruderal species „R”), (Reynolds 1988, 1993; Weithoff et al. 2000). In 1995, Reynolds extended the adaptation strategy of Grime with the subcategory - SS-, within which there were included species tolerant of continuously changing habitat conditions, which develop well in highly stressful conditions, e.g. temporal shortage of biogenic substances or sunlight deficiency. The subcategory - SS- consists mainly of picoplankton of tropical seas and oligotrophic lakes.

It seems that the theory of smooth transition of a population from one life strategy to another one - the most favourable in a given situation $(\mathrm{r} \rightarrow \mathrm{K})$, proved to be correct in relation to lower plants, in this case - algae, and therefore it was applied in the this paper.

Specific conditions prevailing in raised bogs impose specific reactions upon inhabiting there organisms. Therefore many algae species of peat bogs demonstrate exceptional flexibility, which enables their adaptation to changes of environmental factors.

\section{The study area}

The water and peat bog nature reserve „Dury” (Fig. 1) consists of five separate objects situated within a short distance from each other in the forest administration region of Stara Rzeka, the Forest Division of Osie, in the Wdecki Landscape Park (Poland). Waters of these lakes are humotrophic, with small transparency and inconsiderable biological production. Humus compounds contained in the water are responsible for its characteristic yellow-dark brown colour. They also significantly influence the physicochemical factors (Wojciechowski 1987, 1999; Choiński 1995).

The studied objects denoted as Dury 1 and Dury 2, are small dystrophic lakes without any outflow, surrounded by a strip of peat bogs, several to several dozen metres wide. Peat vegetation around the lake forms two distinct zones: a zone of the waterside peat bog without a tussock structure and a peat bog zone with remarkably tussock character, situated further away from the water (Kępczyński 1950; Walas 1950; Nienartowicz et al. 1988, Boiński 1999).

\section{Material and study methods}

Sample collection sites were located according to different types of microhabitats connected with a mosaic structure of Sphagnum mats characteristic of transition mires and raised bogs (Fig. 2). Small valleys, temporary flooded with water, occur here alternately with tussocks of peat mosses grown over with higher vegetation.

Sites at both study places were located along the transect running from the water level to the marshy coniferous forest. At the peat bog Dury 1, with the Sphagnum floating mat ca. $28 \mathrm{~m}$ wide, the first site was located on the borderline between the water surface and the Sphagnum floating mat. The second site was located at a distance ca. $6 \mathrm{~m}$ from the water level in a tussock of peat mosses exposed to frequent shrivelling. The third site was delimited in a water-logged valley, just before a line of trees (mainly pine and European white birch), ca. $18 \mathrm{~m}$ from the water surface. The fourth site was delimited in a small valley surrounded by tussocks of cotton grasses, sedges and young pine trees, within a vegetation fringe, which constitutes a borderline between a Sphagnum mat and marshy pine forest at a distance of more than 20 metres from the water surface.

At the second peat bog (ca. $12 \mathrm{~m}$ wide), the first site was located at the borderline between the water surface and the Sphagnum floating mat. The second one was located at a distance of $3 \mathrm{~m}$ from a small water body on a tussock of peat mosses overgrown with cranberry. The third one was located in a small valley, often flooded with water, in the area overgrown with marsh tea, at a distance of $5 \mathrm{~m}$ from the water. The fourth site was located in the outer, shriv- 


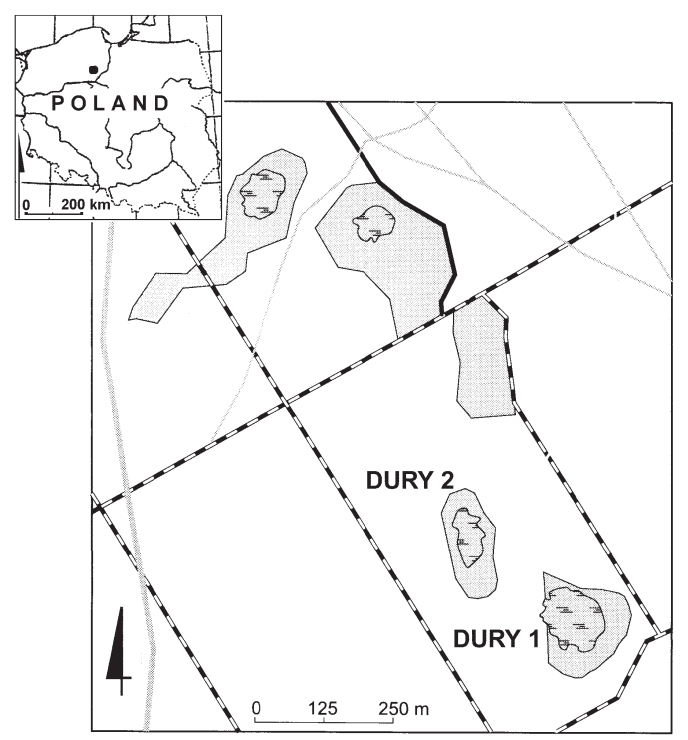

Figure 1. The „Dury” Reserve location in Poland on the Wda River Landscape Park area

elled part of the Sphagnum mat, at a distance of ca. $8 \mathrm{~m}$, at the borderline with marshy pine forest.

Collection of samples was done every two months, from February 1998 till April 1999. For quantitative studies, samples were collected by cutting out some fragments of a peat bog by means of one side sharp cylinder with the surface area of $16 \mathrm{~cm}^{2}$, so as to preserve the water present between peat mosses. Each time at all delimited sites, the following parameters were measured: $\mathrm{pH}$, electrolytic conductivity and the temperature of water stored between peat mosses. Also the relation between the mass of live and dead peat mosses was determined in a studied sample. Also aquarium cultures of peat-bog algae were prepared. These cultures enabled to identify algae species and to observe their ontogenesis.

Species of algae were identified in the laboratory and then counted in sedimentation chambers with the capacity of 5 and $10 \mathrm{ml}$ under an inverted microscope with a magnification of 100 and $400 \mathrm{x}$, in two perpendicular strips. Three replicates from each sample were performed. The average number of specimens was calculated per $10 \mathrm{~cm}^{2}$ of the peat bog area. In order to calculate the average volume of a cell, each characteristic, i.e. the length, the width and the thickness of a cell were measured ca. 50 times. Therefore, the entire morphological variability of the studied taxa was included. Chemical analyses included the concentration of: $\mathrm{P}-\mathrm{PO}_{4}, \mathrm{~N}-\mathrm{NO}_{3}$ and $\mathrm{N}-\mathrm{NH}_{4}$. The concentration of phosphate phosphorus was determined by the molybdate method, the concentration of nitrate nitrogen - by the colorimetric method with sodium salicylate, ammonia nitrogen - by the Nessler method (Hermanowicz et al. 1999).

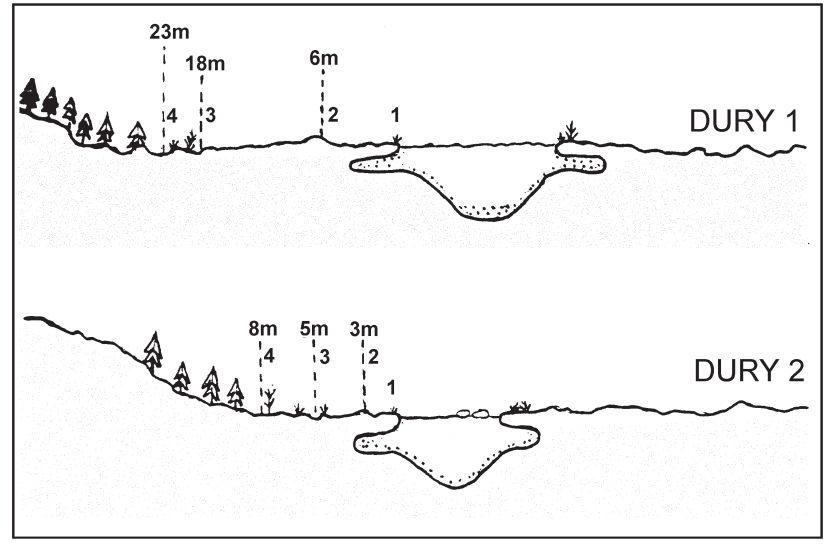

Figure 2. Location of stands within peatbogs - distance from water surface. 1, 2, 3, 4- stands

When analysing the results, statistical methods were applied, using the software Excel 5.0. In order to determine the relationships between the species composition of samples and selected environmental variables, the method of Canonical Correspondence Analysis was applied from the package of statistical procedures MVSP 3.1b (Kovach 1993; Palmer 1998; Van Der Maarel 1998).

\section{The research results}

\subsection{Physicochemical properties of water}

Physical and chemical properties of water from both peat bogs are presented in Table 1. As the distance from the water surface increased, $\mathrm{pH}$ decreased at both studied peat bogs, from 6.3 at the borderline water-peat bog to 3.4 at the borderline peat bog-marshy pine forest. In general, electrolytic conductivity was low. It slightly increased as the distance from the water surface increased, towards the marshy coniferous forest. The concentration of $\mathrm{P}_{-} \mathrm{PO}_{4}$ in the water accumulated in spring between peat mosses was low. It slightly increased during the summer months, reaching the maximum value at the third site in the peat bog Dury 1 . The lowest concentrations of $\mathrm{N}-\mathrm{NO}_{3}$ and $\mathrm{N}-\mathrm{NH}_{4}$ were recorded at the sites situated near the water surface. As the distance from the water surface increased, the concentration of both nitrogen forms at particular sites increased, reaching the highest values in the outer, most dried up part of both peat bogs. However, despite the fact that the values are very low, these are not the values limiting the occur- 


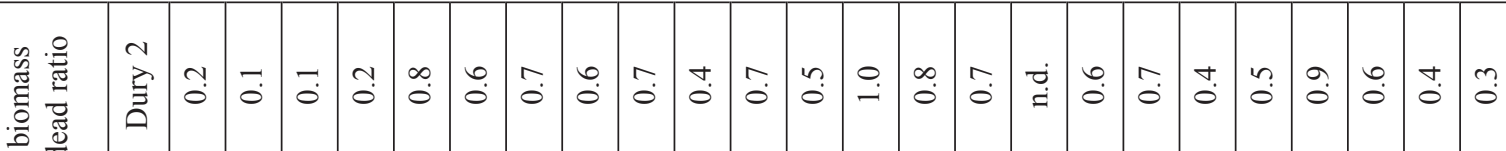

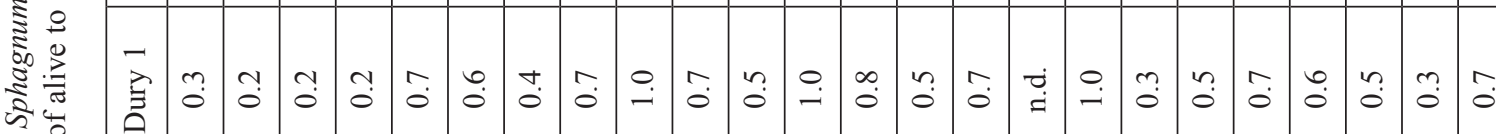

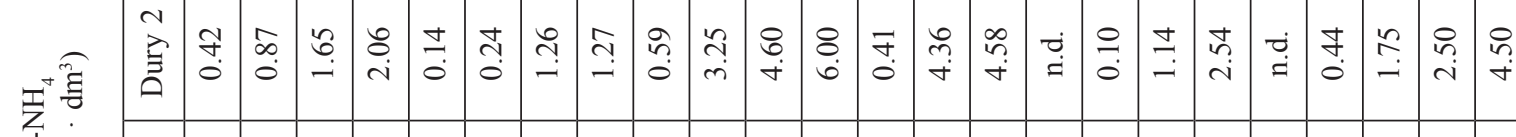

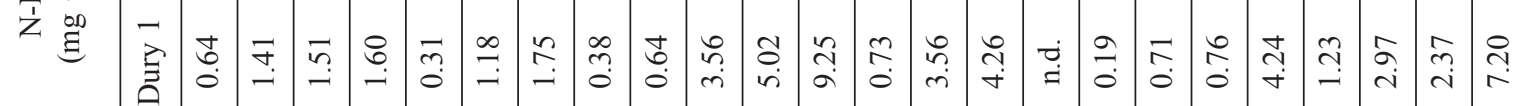

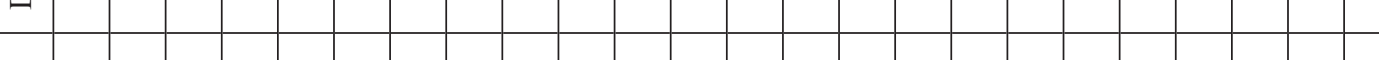

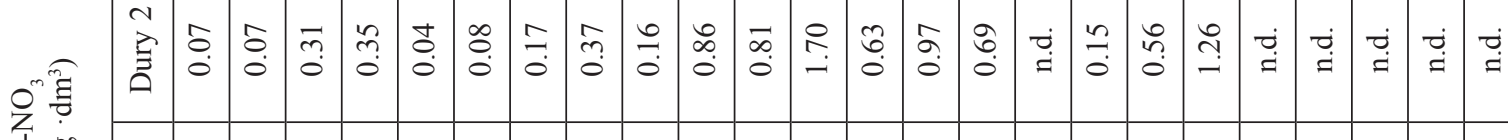

之

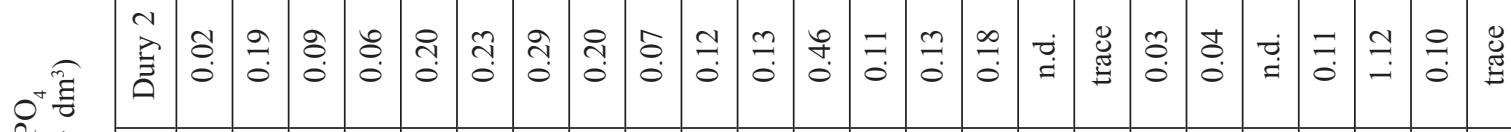

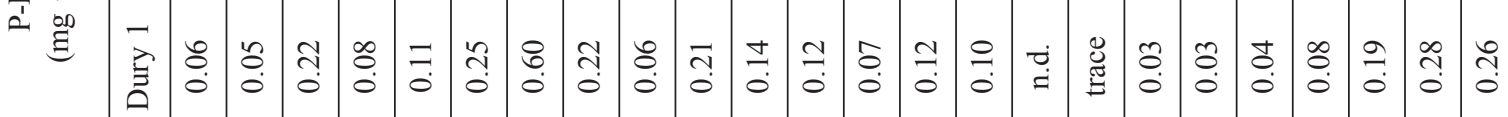

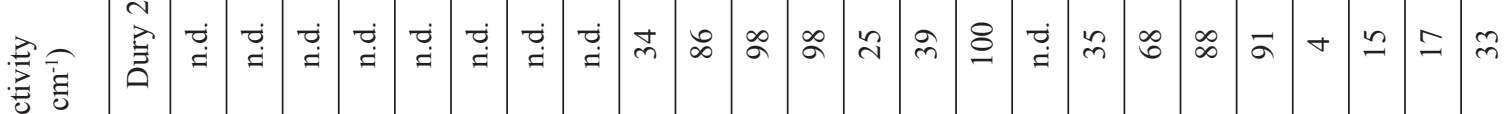

ठ己

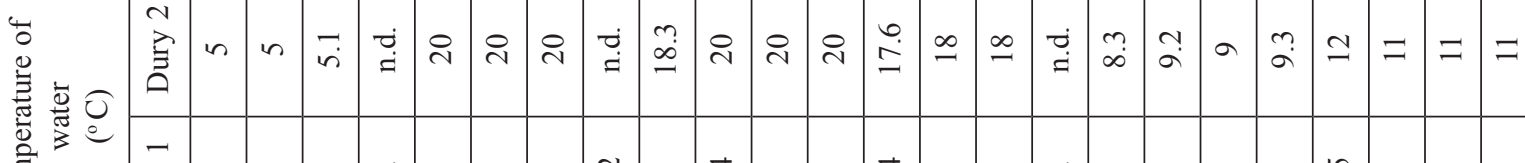

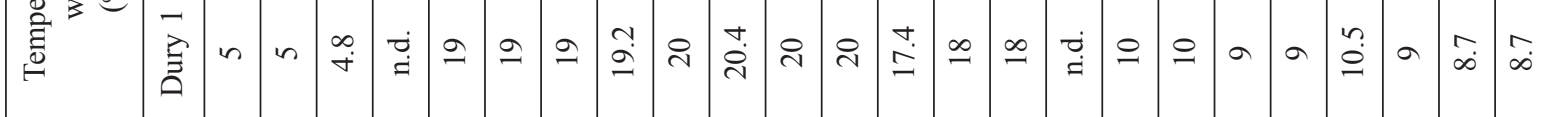

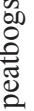

空

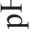

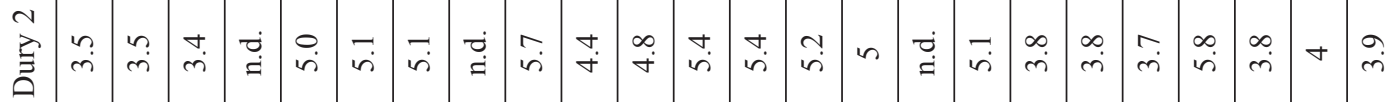

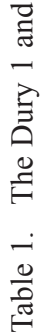

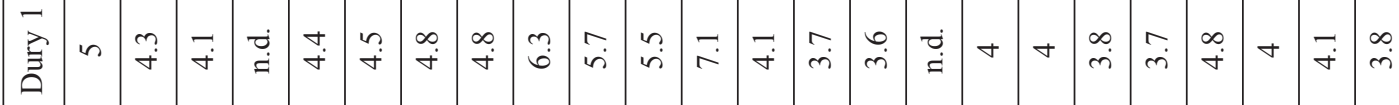

$\stackrel{g}{E}$

$\frac{0}{\frac{0}{\sigma}}$

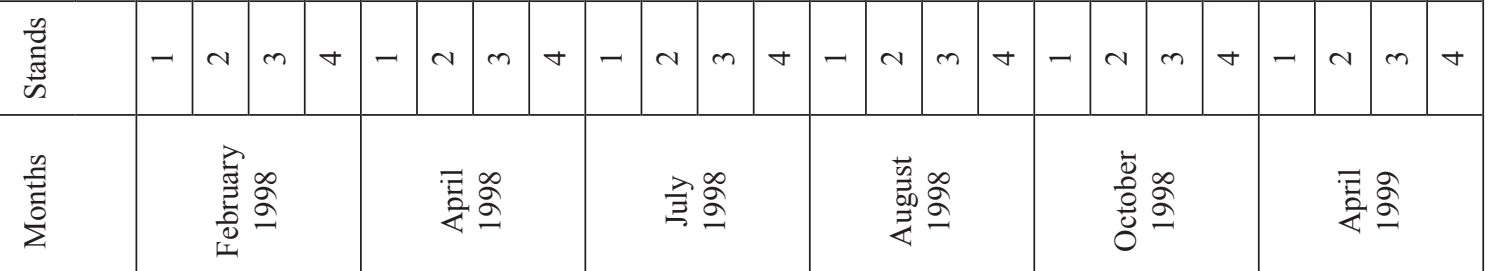


rence and the growth of algae (Oleksowicz 1987; Kawecka \& Eloranta 1994; Matuła 1995).

\subsection{Autecology of selected algae species}

Species for autecological studies were selected based on the count of a given population and its presence in specific microhabitats (Jacuńska \& Soska 2002). Therefore, apart from forms co-dominating at all studied sites along the transects, such as Penium silvae-nigrae f. parallelum or Anisonema ovale, there were also selected species, which occurred only at one or two sites during certain months of the research, e.g. Tetmemorus brebissonii var. brebissonii occurring in summer, or Chroococcus turgidus connected with the sites situated near the water table.

\subsubsection{Penium silvae-nigrae Rabanus f. parallelum W. Krieger 1935}

Penium silvae-nigrae f. parallelum (Fig. 3.) - from the order Desmidiales; it is characterized as a sphagno- and acidophilous species, tolerant of $\mathrm{pH}$ within the range from 3.5 to 5.0, typical of oligotrophic raised bogs, Sphagnum wetlands, sometimes behaving as a subaerophyte. This species also occurs on wet mosses on rocks. The described alga has a wide geographic range, which includes: Europe, Asia and North America. Penium silvae-nigrae f. parallelum is characteristic of extremely acid habitats. In respect of the presence of nitrogen forms available for organisms, it is included within the group of the so-called nitratebionts, for which nitrates are the only assimilable nitrogen form on peat bogs (Kossinskaja 1960; Prescott et al. 1975; Hosiaisluoma 1975; Růžička 1981; Matuła 1995).

It is a large autotroph with a slowly growing cell. In the studied peat bogs, it used to be found both in the habitat highly hydrated (site 1), but also in the environment constantly exposed to overdrying (site 4), in other words, most probably in the conditions of the increased stress for other sphagno- and acidophilous Zygnematales. However, it occurred in the largest numbers at the sites situated near the water surface. As the distance from the water increased, its count decreased (Tab. 2). The volume of individual cells was changing slightly: from $22,000-28,000 \mu \mathrm{m}^{3}$ at site 1 to $22,000-26,000 \mu^{3}$ at site 4 . Specimens of this species were tolerant of fluctuations in the water reaction within the $\mathrm{pH}$ range of 3.5-6.0, however they reached the highest count with $\mathrm{pH}$ below 5. Large dimensions of cells and the considerable count, proves the species preferences for low concentrations of biogens. On both peat bogs this species occurred both during the summer and late autumn months, but also in winter months.

Based on the performed analyses, one can state that the population of Penium silvae-nigrae f. parallelum has a wide range of tolerance, first of all, to strong fluctua-
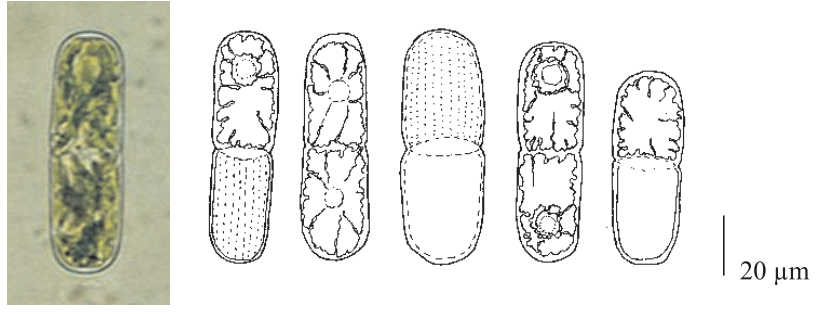

Figure 3. Penium silvae-nigrae Rabanus f. parallelum W. Krieger 1935

tions of the water level along the transects, but also to significant changes in electrolytic conductivity and $\mathrm{pH}$. This taxon reached its quantitative and qualitative optimum in different, sometimes extreme habitat conditions. This phenomenon can be accounted for by the „K strategy” of adaptation (Luścińska \& Jakubowska 1999; Jacuńska \& Soska 2002), according to which, organisms representing this type of strategy, consume the energy „economically" and slowly. It enables the specimens to grow and develop slowly. The count of populations, which develop this way, is more or less balanced over a longer period of time, and the multiplication process is relatively slower.

\subsubsection{Anisonema ovale Klebs 1893}

Anisonema ovale (Fig. 4.) - a colourless, heterotrophic euglenoid, occurring in stagnant and slow-moving waters, in pools, ditches, in polluted waters, $\alpha-\beta$ mesosaprobic. It is also encountered in ponds and in sediments of shallow water bodies. It is a cosmopolitan species, quoted from Europe, Asia, Africa, North and South America (Starmach 1983; Wołowski 1998). So far, the species was not recorded on raised bogs.

Anisonema ovale co-dominated in all distinguished microhabitats with the above described species of Penium. However, contrary to Penium, the increased count of this taxon was observed together with the increased distance from the water surface towards the marshy coniferous forest (Tab. 2). The largest numbers of this species occurred at site 3 in April 1998, reaching the number of 4,000,000 specimens per $\mathrm{dm}^{2}$ of the surface area of the peat bog Dury 2. In the summer, the count of Anisonema dropped to $1,050,000$ specimens and in February 1998 - to 8,870 35,000 specimens per $\mathrm{dm}^{2}$ of the studied surface area of the peat bog. Together with the increased count, smaller dimensions of specimens were observed: from $616 \mu^{3}$ in February to $315 \mu^{3}$ in April 1998. Most probably, it was related to rapid multiplication of this species in spring, through exploitation of the relative ,abundance” of organic 


\begin{tabular}{|c|c|c|c|c|c|c|c|c|c|c|c|c|c|c|c|c|c|c|c|c|c|c|c|c|c|c|}
\hline \multirow{4}{*}{ 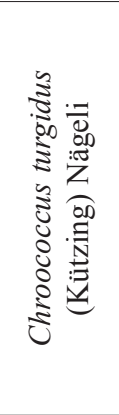 } & g & 总 & & 1 & 1 & & $\frac{\sqrt{2}}{\frac{3}{2}}$ & 1 & 1 & & $\begin{array}{l}m \\
\infty \\
m \\
m\end{array}$ & 1 & & & $\underset{\frac{9}{1}}{n}$ & 1 & 1 & & $\begin{array}{l}m \\
\infty \\
m \\
m\end{array}$ & 1 & 1 & 1 & 1 & 1 & & \\
\hline & $\frac{z}{9}$ & $\overrightarrow{\mathrm{E}}$ & & 1 & 1 & 1 & 1 & 1 & 1 & 1 & 1 & 1 & 1 & 1 & $\begin{array}{l}m \\
\infty \\
\infty \\
\end{array}$ & 1 & 1 & 1 & ते & 1 & 1 & 1 & 1 & & 1 & 1 \\
\hline & 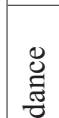 & $\begin{array}{l}\sim \\
\stackrel{2}{\partial} \\
\partial\end{array}$ & & 1 & 1 & 1 & $\begin{array}{l}\overrightarrow{5} \\
\text { oे } \\
\text { के }\end{array}$ & 1 & 1 & 1 & $\begin{array}{l}8 \\
\infty \\
\text { N } \\
\text { N }\end{array}$ & 1 & 1 & 1 & 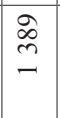 & 1 & 1 & 1 & 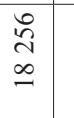 & 1 & 1 & 1 & 1 & 1 & 1 & 1 \\
\hline & 言 & $\overrightarrow{\mathrm{Z}}$ & & 1 & 1 & 1 & 1 & 1 & 1 & 1 & 1 & 1 & 1 & 1 & $\overline{\overparen{I}}$ & 1 & 1 & 1 & के & 1 & 1 & 1 & 1 & 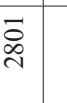 & 1 & 1 \\
\hline \multirow{4}{*}{ 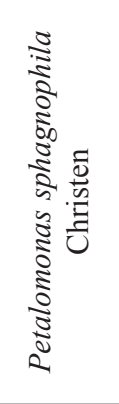 } & $\cong$ & 胥 & & 1 & \begin{tabular}{|c|}
$\tilde{c}$ \\
$\infty$ \\
$\infty$ \\
$\sim$
\end{tabular} & 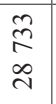 & 1 & $\begin{array}{l}\infty \\
\vdots \\
\vdots \\
\end{array}$ & \begin{tabular}{|l|}
$\infty$ \\
0 \\
$\vdots$ \\
\\
\end{tabular} & $\begin{array}{l}\stackrel{\Xi}{\Xi} \\
\underset{\sim}{J}\end{array}$ & \begin{tabular}{|c|}
$\tilde{c}$ \\
$\infty$ \\
$\infty$ \\
$\sim$
\end{tabular} & \begin{tabular}{|c|}
$\tilde{c}$ \\
$\tilde{N}$ \\
$\infty$ \\
$\tilde{N}$
\end{tabular} & 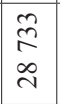 & 1 & $\begin{array}{l}\tilde{N} \\
0 \\
d \\
d\end{array}$ & $\begin{array}{l}\stackrel{N}{m} \\
\stackrel{\sim}{d}\end{array}$ & 1 & 1 & 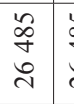 & 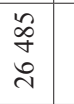 & 1 & 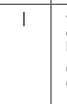 & & 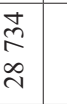 & & \\
\hline & $\stackrel{3}{\circ}$ & $\overrightarrow{\mathrm{z}}$ & & 1 & 1 & 1 & $\begin{array}{l}\mathscr{\infty} \\
\stackrel{\tilde{m}}{ \pm}\end{array}$ & 1 & 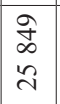 & $\begin{array}{l}\text { 文 } \\
\infty \\
\grave{\lambda}\end{array}$ & \begin{tabular}{|l}
$\mid \vec{d}$ \\
dे \\
d
\end{tabular} & 1 & $\begin{array}{l}\mid \vec{d} \\
\text { dे } \\
\text { dे }\end{array}$ & $\begin{array}{l}\vec{d} \\
\vec{\nabla} \\
\vec{\nabla}\end{array}$ & 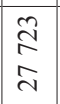 & $\begin{array}{l}\stackrel{2}{i} \\
\stackrel{\Delta}{\lambda}\end{array}$ & 1 & 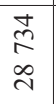 & \begin{tabular}{l|l}
1 \\
\end{tabular} & 1 & 1 & 1 & & $\begin{array}{l}\text { d } \\
\text { d } \\
\text { i }\end{array}$ & 1 & I \\
\hline & $\begin{array}{l}\text { ठ্ } \\
\text { E్ }\end{array}$ & 胥 & 1 & 1 & \begin{tabular}{l}
\multirow{2}{*}{} \\
$\sigma$ \\
$\sigma$
\end{tabular} & $\stackrel{\ominus}{\exists}$ & 1 & $\begin{array}{l}\hat{z} \\
\bar{v}\end{array}$ & 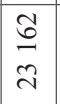 & $\begin{array}{l}n \\
\tilde{q} \\
n \\
n\end{array}$ & \begin{tabular}{|l|} 
\\
0 \\
0 \\
0 \\
0
\end{tabular} & $\begin{array}{l}8 \\
\infty \\
0 \\
\text { v }\end{array}$ & $\begin{array}{l}\overrightarrow{\bar{N}} \\
\vec{\nabla}\end{array}$ & 1 & $\overrightarrow{\widetilde{N}}$ & \% & 1 & 1 & \begin{tabular}{c|}
$\begin{array}{c}\tilde{\infty} \\
\tilde{\infty} \\
a\end{array}$ \\
\end{tabular} & $\begin{array}{l}\stackrel{a}{\infty} \\
\stackrel{m}{-}\end{array}$ & 1 & 1 & $\underset{\substack{N \\
i}}{i}$ & 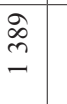 & 1 & 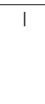 \\
\hline & 言 & $\overrightarrow{\mathrm{z}}$ & ' & 1 & 1 & 1 & $\begin{array}{l}\frac{1}{2} \\
6 \\
6\end{array}$ & 1 & 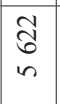 & 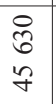 & $\begin{array}{l}\infty \\
\sigma \\
= \\
=\end{array}$ & 1 & $\begin{array}{l}\text { तु } \\
\text { in }\end{array}$ & $\begin{array}{l}\overrightarrow{\mathrm{N}} \\
\vec{\sigma}\end{array}$ & 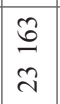 & 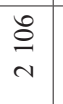 & 1 & के & 1 & 1 & 1 & 1 & $\begin{array}{l}\text { त्र } \\
\text { in }\end{array}$ & 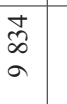 & 1 & 1 \\
\hline \multirow{4}{*}{ 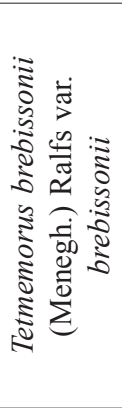 } & 品 & 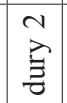 & 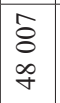 & 1 & 1 & 1 & $\begin{array}{l}\tilde{\sigma} \\
\substack{\sigma \\
\alpha}\end{array}$ & 1 & 1 & 1 & 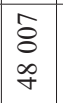 & $\begin{array}{l}\hat{8} \\
\infty \\
\alpha \\
\alpha\end{array}$ & 1 & 1 & 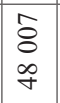 & 1 & 1 & 1 & $\begin{array}{l}\widehat{\widehat{I}} \\
o \\
q\end{array}$ & 1 & 1 & 1 & $\begin{array}{l}\text { fy } \\
\text { in } \\
\text { în }\end{array}$ & 1 & 1 & 1 \\
\hline & $\frac{3}{3}$ & $\overrightarrow{\mathrm{C}}$ & ' & 1 & 1 & 1 & \begin{tabular}{|l} 
\\
8 \\
$o$ \\
$o$
\end{tabular} & 1 & 1 & 1 & $\begin{array}{l} \\
8 \\
o \\
o \\
\alpha\end{array}$ & $\begin{array}{l} \\
8 \\
o \\
o \\
q\end{array}$ & 1 & 1 & $\begin{array}{l}\infty \\
\infty \\
\infty \\
f \\
\forall\end{array}$ & $\begin{array}{l}\infty \\
\infty \\
\infty \\
\forall\end{array}$ & 1 & 1 & 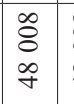 & $\begin{array}{l}\infty \\
\stackrel{8}{ } \\
\infty \\
\infty\end{array}$ & 1 & 1 & 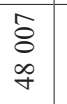 & $\begin{array}{l}\hat{\sigma} \\
\infty \\
\infty \\
\alpha\end{array}$ & 1 & 1 \\
\hline & \begin{tabular}{|l} 
\\
\\
E్ \\
]
\end{tabular} & 胥 & 1 & 1 & 1 & 1 & $\stackrel{8}{!}$ & 1 & 1 & 1 & 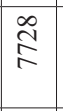 & 嵒 & 1 & 1 & $\frac{8}{2}$ & 1 & 1 & 1 & $\stackrel{\Xi}{\Xi}$ & 1 & 1 & 1 & $\underset{\substack{\infty\\
}}{n}$ & 1 & 1 & 1 \\
\hline & 言 & $\overrightarrow{\mathrm{g}}$ & 1 & 1 & 1 & 1 & ڤి & 1 & 1 & 1 & o & is & 1 & 1 & 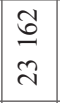 & 年 & 1 & 1 & ถ & 恕 & 1 & 1 & $\begin{array}{l}\stackrel{8}{\circ} \\
+\end{array}$ & $\begin{array}{l}\tilde{\delta} \\
\infty \\
\lambda \\
\hat{n}\end{array}$ & 1 & 1 \\
\hline \multirow{4}{*}{ 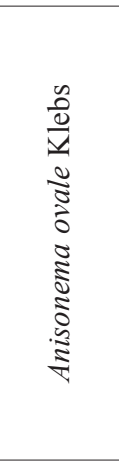 } & $\stackrel{\Xi}{\Xi}$ & $\begin{array}{l}2 \\
2 \\
z \\
z\end{array}$ & ờ & ơ & 1 & ós & $\frac{\partial}{m}$ & $\frac{n}{m}$ & $\begin{array}{l}n \\
\infty \\
\infty\end{array}$ & $\hat{q}$ & 京 & 离 & 1 & $\stackrel{\infty}{m}$ & $\overrightarrow{\widetilde{\gamma}}$ & $\vec{q}$ & 1 & 1 & \begin{tabular}{l|l}
$\infty$ \\
$\infty$ \\
$\infty$
\end{tabular} & 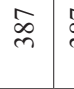 & 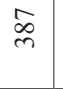 & $\underset{\infty}{\infty}$ & $\bar{F}$ & $\begin{array}{ll}0 \\
+\end{array}$ & $\vec{n}$ & in \\
\hline & $\frac{z}{\circ}$ & $\overrightarrow{\mathrm{E}}$ & $\frac{0}{6}$ & $\frac{6}{6}$ & 1 & 1 & $m$ & बे & ह్లి & లెm & $\mid \begin{array}{c}\infty \\
\stackrel{f}{f}\end{array}$ & $\stackrel{\infty}{\infty}$ & $\stackrel{\infty}{\infty}$ & $\hat{b}$ & 胥 & F & $\stackrel{\infty}{\%}$ & $\stackrel{\infty}{ซ}$ & 常 & 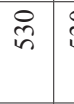 & in & in & is & $\frac{0}{6}$ & $\stackrel{0}{0}$ & $\frac{0}{6}$ \\
\hline & 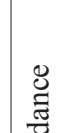 & $\stackrel{\sim}{\stackrel{2}{z}}$ & $\begin{array}{l}\hat{n} \\
\hat{n}\end{array}$ & $\begin{array}{l}\tilde{\omega} \\
\infty \\
\sigma\end{array}$ & 1 & $\begin{array}{l}\frac{a}{5} \\
\text { m } \\
m\end{array}$ & \begin{tabular}{|l|}
$\infty$ \\
0 \\
0 \\
0 \\
0 \\
$n$
\end{tabular} & $\begin{array}{l}\stackrel{2}{2} \\
\vec{n} \\
\vec{\infty}\end{array}$ & 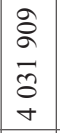 & 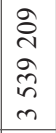 & $\begin{array}{l}P \\
\infty \\
\infty \\
\infty\end{array}$ & $\begin{array}{l}\text { ते } \\
\infty \\
0 \\
0\end{array}$ & 1 & 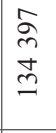 & $\mid \begin{array}{l}\hat{\sigma} \\
\tilde{\alpha} \\
\infty \\
=\end{array}$ & $\begin{array}{l}0 \\
\infty \\
\infty \\
\infty\end{array}$ & 1 & 1 & 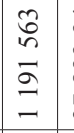 & 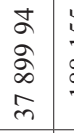 & $\begin{array}{l}n \\
\stackrel{n}{n} \\
\infty \\
\infty \\
\sim\end{array}$ & $\begin{array}{l}\frac{9}{5} \\
=\end{array}$ & 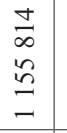 & \begin{tabular}{l|l}
$\infty$ & \\
$\infty$ & \\
$\infty$ & \\
$\infty$ & \\
$\alpha$ &
\end{tabular} & $\begin{array}{l}\tilde{b} \\
\tilde{D} \\
\stackrel{5}{1}\end{array}$ & $\begin{array}{l}\frac{g}{i} \\
i \\
m\end{array}$ \\
\hline & 言 & $\overrightarrow{\mathrm{E}}$ & $\begin{array}{l}\text { ga } \\
\text { in }\end{array}$ & $\begin{array}{l}\stackrel{R}{\infty} \\
\infty \\
\infty\end{array}$ & 1 & 1 & 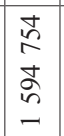 & $\begin{array}{l}\infty \\
\infty \\
\infty \\
\\
\\
\end{array}$ & 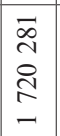 & 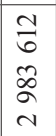 & 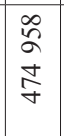 & 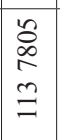 & $\begin{array}{l}\stackrel{a}{\infty} \\
\infty\end{array}$ & 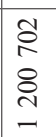 & \begin{tabular}{|l|}
$\vec{J}$ \\
\\
0 \\
$\infty$
\end{tabular} & 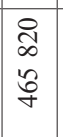 & 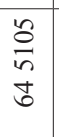 & 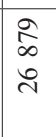 & \begin{tabular}{|l|}
\multirow{2}{*}{} \\
$\stackrel{2}{2}$ \\
2 \\
2
\end{tabular} & $\begin{array}{l:l}2 & \\
\infty & \\
\infty & \vdots\end{array}$ & 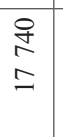 & $\begin{array}{l}0 \\
\text { ch } \\
c \\
m\end{array}$ & $\begin{array}{l}\approx \\
\tilde{i} \\
\tilde{b} \\
\tilde{6}\end{array}$ & $\begin{array}{l}\infty \\
\stackrel{\alpha}{a} \\
\vec{\sigma}\end{array}$ & $\begin{array}{c}\infty \\
\infty \\
\infty \\
\neq\end{array}$ & $\begin{array}{l}\stackrel{े}{o} \\
\infty \\
\infty\end{array}$ \\
\hline \multirow{4}{*}{ 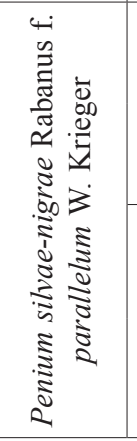 } & 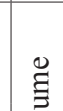 & 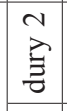 & \begin{tabular}{|l|}
\multirow{2}{*}{} \\
2 \\
$i$ \\
$i$ \\
\end{tabular} & $\begin{array}{l}\tilde{y} \\
i \\
\sim \\
\end{array}$ & \begin{tabular}{|l|}
$\tilde{n}$ \\
$i$ \\
$\sim$ \\
$n$
\end{tabular} & $\begin{array}{l}\tilde{y} \\
\hat{y} \\
\hat{n}\end{array}$ & $\begin{array}{l}\mid \vec{a} \\
\vec{\lambda} \\
\vec{\lambda}\end{array}$ & \begin{tabular}{|l|} 
\\
$i$ \\
$n$ \\
$n$ \\
$n$
\end{tabular} & 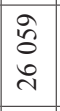 & 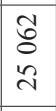 & $\begin{array}{l}q \\
\\
\vec{n}\end{array}$ & 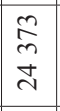 & \begin{tabular}{|l|}
$\hat{i}$ \\
$\tilde{n}$ \\
\end{tabular} & $\begin{array}{l}\hat{\tilde{n}} \\
\tilde{n} \\
\text { त } \\
\end{array}$ & 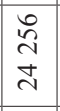 & 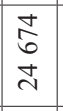 & 1 & 1 & \begin{tabular}{|l|}
$\hat{\tilde{n}}$ \\
$\tilde{i}$ \\
\end{tabular} & 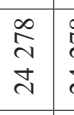 & 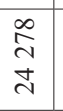 & 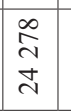 & \begin{tabular}{|l|}
$\hat{n}$ \\
$i$ \\
$\dot{d}$
\end{tabular} & \begin{tabular}{l}
$\infty$ \\
\multirow{2}{*}{} \\
$\tilde{\lambda}$ \\
$\tilde{\lambda}$
\end{tabular} & $\begin{array}{l}n \\
\hat{b} \\
\vdots \\
\text { d. }\end{array}$ & 1 \\
\hline & $\frac{5}{3}$ & $\begin{array}{l}\vec{Z} \\
\vec{\Xi} \\
\vec{z}\end{array}$ & 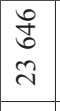 & \begin{tabular}{|l|}
\multirow{2}{*}{} \\
\\
\multirow{2}{*}{} \\
\\
\end{tabular} & 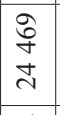 & $\begin{array}{l}8 \\
\text { ò } \\
\text { ḋ } \\
\text { d } \\
\end{array}$ & \begin{tabular}{|l|}
\multirow{2}{*}{} \\
\multirow{2}{*}{} \\
\\
\end{tabular} & $\begin{array}{l}0 \\
\infty \\
\tilde{\lambda} \\
\end{array}$ & 1 & $\begin{array}{l}\vec{y} \\
\vec{q} \\
a \\
\end{array}$ & $\begin{array}{l}\stackrel{0}{w} \\
\ddot{i} \\
\end{array}$ & 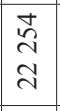 & 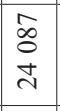 & \begin{tabular}{|l|}
\multirow{2}{*}{} \\
$\tilde{n}$ \\
d
\end{tabular} & $\begin{array}{l}\tilde{N} \\
\tilde{\alpha} \\
\tilde{2}\end{array}$ & 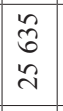 & 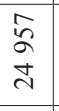 & 1 & 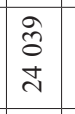 & 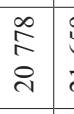 & $\begin{array}{l}\hat{b} \\
\vec{\lambda}\end{array}$ & 1 & \begin{tabular}{|l|}
0 \\
0 \\
\\
d \\
\end{tabular} & 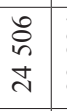 & 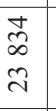 & 1 \\
\hline & $\begin{array}{l}\text { : } \\
\text { 胥 }\end{array}$ & 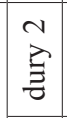 & $\begin{array}{l}\text { N } \\
i \\
\dot{d} \\
\end{array}$ & $\begin{array}{l}\mathcal{N} \\
=\end{array}$ & 궁 & $\begin{array}{l}\text { in } \\
0 \\
\pm \\
\pm\end{array}$ & 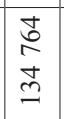 & $\begin{array}{l}\hat{2} \\
\hat{n} \\
n\end{array}$ & $\begin{array}{l}a \\
\frac{a}{b} \\
i n\end{array}$ & $\begin{array}{l}\tilde{\sigma} \\
\tilde{m} \\
\tilde{m}\end{array}$ & $\begin{array}{l}0 \\
\check{R} \\
f \\
f\end{array}$ & $\begin{array}{l}1 \\
0 \\
0 \\
0 \\
0 \\
-1\end{array}$ & $\begin{array}{l}0 \\
0 \\
r\end{array}$ & $\frac{5}{6}$ & \begin{tabular}{|l|} 
\\
\multirow{4}{*}{} \\
$o$ \\
$\alpha$ \\
$\sigma$
\end{tabular} & 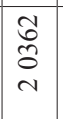 & 1 & 1 & 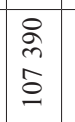 & $\begin{array}{l}\stackrel{n}{\sigma} \\
\dot{q}\end{array}$ & \begin{tabular}{l}
\multirow{2}{*}{} \\
\multirow{\sigma}{*}{}
\end{tabular} & $\begin{array}{l}0 \\
i n \\
m\end{array}$ & \begin{tabular}{|l|} 
\\
$\stackrel{2}{2}$ \\
$\underline{0}$ \\
\end{tabular} & $\begin{array}{l}\text { İ } \\
\text { in }\end{array}$ & $\begin{array}{l}\bar{b} \\
\text { m }\end{array}$ & 1 \\
\hline & 壳 & $\overrightarrow{\mathrm{E}}$ & $\begin{array}{l}8 \\
\infty \\
\sim\end{array}$ & $\begin{array}{l}\vec{n} \\
\infty \\
\sim\end{array}$ & \begin{tabular}{|l|} 
\\
$b$ \\
$b$ \\
$o$ \\
6
\end{tabular} & $\begin{array}{l}0 \\
\infty \\
\infty \\
a\end{array}$ & $\begin{array}{l}n \\
0 \\
0 \\
m\end{array}$ & 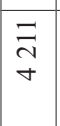 & 1 & $\begin{array}{l}\text { ळू } \\
\text { iे }\end{array}$ & $\Xi$ & \begin{tabular}{|l|}
$\tilde{n}$ \\
$\infty$ \\
$a$
\end{tabular} & $\begin{array}{l}0 \\
0 \\
0 \\
0 \\
0\end{array}$ & $\begin{array}{l}\hat{\alpha} \\
=\end{array}$ & $\begin{array}{l}\text { I } \\
\text { ปे }\end{array}$ & \begin{tabular}{|l|}
$\overrightarrow{0}$ \\
$\infty$ \\
$\infty$ \\
\\
\end{tabular} & $\begin{array}{l}\stackrel{2}{ } \\
\text { ळे }\end{array}$ & 1 & 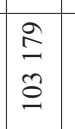 & $\begin{array}{l}\text { ले } \\
\text { mె } \\
\end{array}$ & 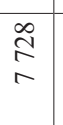 & & 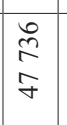 & $\begin{array}{l}\stackrel{N}{\kappa} \\
\bar{n}\end{array}$ & $\begin{array}{l}2 \\
\vdots \\
n \\
m\end{array}$ & \\
\hline \multicolumn{3}{|c|}{ spurtS } & - & $N$ & $m$ & $\theta$ & - & $a$ & $m$ & $\theta$ & - & $n$ & $m$ & $\theta$ & - & 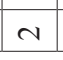 & $n$ & $\nabla$ & - & \begin{tabular}{l|l}
$N$ & 0 \\
\end{tabular} & $n$ & $\theta$ & - & 4 & $m$ & 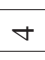 \\
\hline \multicolumn{2}{|c|}{$\begin{array}{l}\text { 言 } \\
\sum^{\circ}\end{array}$} & & \multicolumn{3}{|c|}{$\begin{array}{l}\overrightarrow{0} \\
\tilde{J}_{0}^{\infty} \\
0 \\
0 \\
0 \\
\sigma\end{array}$} & \multicolumn{4}{|c|}{$\begin{array}{l}\overline{\mathbf{z}} \\
\text { 安 }\end{array}$} & \multicolumn{5}{|c|}{ 光 $\stackrel{\infty}{\sigma}$} & \multicolumn{3}{|c|}{ 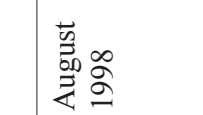 } & \multicolumn{4}{|c|}{$\begin{array}{l}\overline{0} \\
\stackrel{0}{0} \infty \\
\stackrel{0}{0} \\
0\end{array}$} & \multicolumn{4}{|c|}{ 㫣宗 } & \\
\hline
\end{tabular}



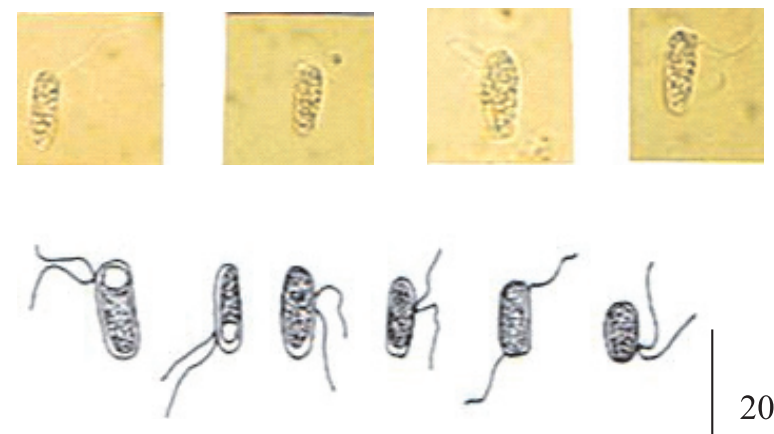

$20 \mu \mathrm{m}$

Figure 4. Anisonema ovale Klebs 1893
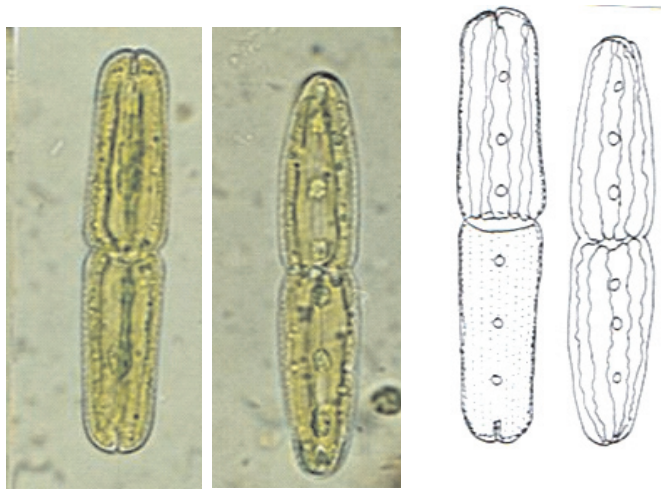

$20 \mu \mathrm{m}$

Figure 5. Tetmemorus brebissonii (Meneghini) Ralfs 1844 var. brebissonii

\subsubsection{Tetmemorus brebissonii (Meneghini) Ralfs 1844 var. brebissonii}

characterized by a short life cycle. Its multiplication proceeds through longitudinal division of a mother cell, so the number of specimens doubles within a short time.

This species was recorded within the $\mathrm{pH}$ range of 3.5 and 6.5. It was more frequent, however, with lower values of the index. Anisonema was tolerant of low concentrations of $\mathrm{P}_{-} \mathrm{PO}_{4}, \mathrm{~N}_{-} \mathrm{NO}_{3}$ and $\mathrm{N}-\mathrm{NH}_{4}$, occurring in large numbers and reaching large dimensions. As it appears, however, from the performed analyses, there are no statistically significant correlations between the size of cells or the count of this species, and the studied physicochemical factors of the habitat. Anisonema ovale occurs in large numbers both at well hydrated sites and those situated along the peat bog margins, where relative water deficiency prevails for most of the year. High resistance of this species to „extreme” overdrying in the given conditions, can be confirmed by the presence of Anisonema in August 1998 at site 4, at the borderline between the Sphagnum mat and the marshy coniferous forest, during the drought prevailing for a month in the Wdecki Landscape Park and the absence of any other algae species. At both studied objects, Anisonema ovale occurred in all the microhabitats during the consecutive months of the research.

Most probably, this small heterotrophic euglenoid is characterized by the „r- strategy" of the development, as compared to large, slowly growing autotrophic desmids copopulating the studied peat bogs. This type of strategy is most frequent in populations of organisms, whose small dimensions are compensated by tremendous reproductiveness and a short cycle of biological development. Metabolism of cellular organisms favouring the $r$ - development strategy enables the process of intensive multiplication within a short time. In this process, organisms use their all energy resources (Burchardt 1999). Therefore, the development of such populations is determined by the habitat richness.

Tetmemorus brebissonii var. brebissonii (Fig. 5.) is a desmid, described as a sphagno and acidophilous taxon. It occurs on peat bogs, in dystrophic lakes near the shoreline zone with Sphagnum sp., in channels and streams, in muddy bends of rivers, between mosses. It tolerates $\mathrm{pH}$ changes within the range from 3.8 to 7.5. It is a cosmopolitan species occurring in Europe, Asia, Africa and North America (Kossinskaja 1960; Růžiččka 1981; Matuła 1995).

In the studied peat bogs, the species found its optimum at the first site situated at the borderline between the water and the floating Sphagnum mat, and the second one - situated in a small valley frequently flooded with water. It occurred most frequently in August 1998 and in April 1999, reaching respectively the number of 23,162 and 23,857 specimens per $\mathrm{dm}^{2}$ of the peat bog surface area (Tab. 2). In autumn, the number of specimens was decreasing to 11,200 at the first site of the peat bog Dury 2 and 690 at the corresponding site of the peat bog Dury 1 . Together with the increase in the number of specimens of Tetmemorus brebissonii, there were not much changes observed in the size of cells of this species. Irrespective of the season, the average volume of a cell ranged from 45,000 to 52,500 $\mu \mathrm{m}^{3}$. At both peat bogs, the species occurred within the $\mathrm{pH}$ range of 3.5-6.5, reaching higher numbers with lower values of this parameter $\left(23,000\right.$ specimens per $\mathrm{dm}^{2}$ of the surface area with $\mathrm{pH}=4.0$ ). However, the positive correlation $(r=+0.49)$ was found between the average volume of a cell of Tetmemorus and the $\mathrm{pH}$ value, i.e. together with increase of $\mathrm{pH}$, dimensions of cells were slightly increasing.

Also the negative correlation was found $(r=-0.37)$ between the values of electrolytic conductivity and the number of Tetmemorus specimens. Also the negative correlation was revealed $(r=-0.53)$ between the count of this 
species and the increase of the nitrogen nitrate content in the water. With higher concentrations of this biogen in the water, the count of Tetmemorus specimens evidently decreased, from 23000 specimens per unit area with $\mathrm{N}-\mathrm{NO}_{3}$ equal to $0.1 \mu \mathrm{g}$ per $\mathrm{dm}^{3}$ of water, to 500 specimens with the increased concentration of $\mathrm{N}_{-} \mathrm{NO}_{3}$ to $1.0 \mu \mathrm{g}$ per $\mathrm{dm}^{3}$ of water.

Tetmemorus brebissonii var. brebissonii demonstrates an obvious affinity to highly hydrated habitats - it was found only at the sites situated at the borderline between the water and the Sphagnum mat, as well as in small depressions permanently filled with water. It appears to be not resistant to overdrying and it was not encountered at other sites, distant from the water surface.

Most probably, Tetmemorus brebissonii var. brebissonii, similarly to other large, autotrophic desmids, represents the $\mathrm{K}$ - strategy of development, i.e. the slow growth and the development of an organism in the environment with high deficiency of main nutrients and strong competition (Reynolds 1988; Sommer 1981, 1988; Kawecka \& Eloranta 1.c).

\subsubsection{Petalomonas sphagnophila Christen 1962}

Petalomonas sphagnophila (Fig. 6.) - a colourless, heterotrophic euglenoid, occurring in stagnant and slow-moving waters, on the raised bogs, especially between Sphagnum spp. It occurs in the northern part of Europe; so far it was not reported from other parts of the world (Starmach 1983). There is not much data on requirements or environmental adaptations of this species.

In the studied peat bogs, Petalomonas sphagnophila occurred along transects in all microhabitats. The highest frequency was recorded in April 1998, when 45630 specimens were recorded per $\mathrm{dm}^{2}$ of the surface area of the peat bog (Tab. 2). The lowest count was recorded in August $1998-695$ specimens per $\mathrm{dm}^{2}$. The species tolerates fluctuations of the $\mathrm{pH}$ index within the range from 3.5 to 7.5 , however it occurred most frequently within the range of 4.5-5.0. Together with the increased $\mathrm{pH}$ index, an obvious drop in the average volume of a cell was recorded (negative correlation $r=-0.62$ ), from 34,000 $\mu^{3}$ with $\mathrm{pH}$ of 5.0 , to $22,500 \mu \mathrm{m}^{3}$ with $\mathrm{pH}$ of $6.5-7.5$. It appears from the above that the studied species is a typical sphagno- and acidophilous species, reaching its quantitative and qualitative optimum with low water reaction. Together with the increasing distance from the water surface, the count of species slightly increased at particular sites; the average size of a cell fluctuated within the range from $23,000-34,500 \mu \mathrm{m}^{3}$ at sites 1 and 2, to 23,000-28,500 $\mu \mathrm{m}^{3}$ at sites 3 and 4. Petalomonas sphagnophila largely tolerated changes in the values of electrolytic conductivity, from 10 at site 1 , to $154 \mu \mathrm{S} \cdot \mathrm{cm}^{1}$ at site 4 . However, the negative correlation was recorded $(r=-0.44)$ between the

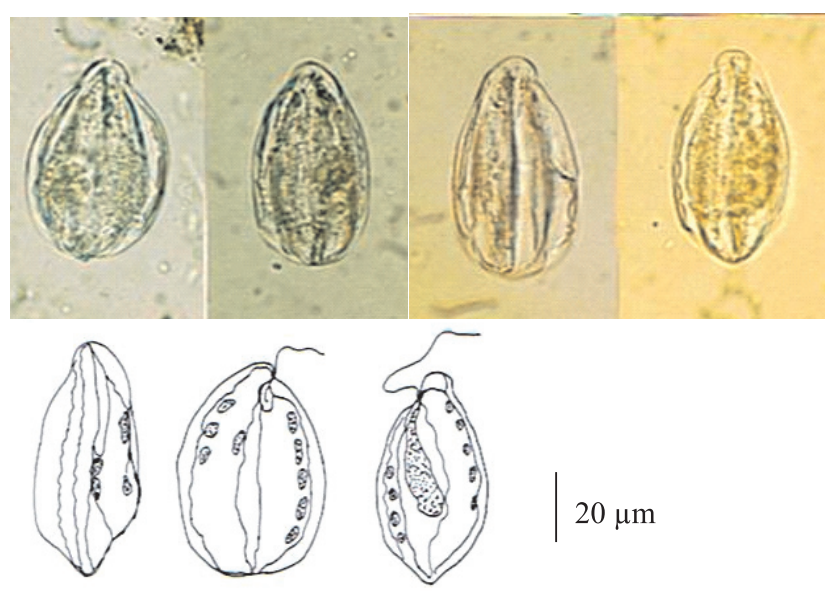

Figure 6. Petalomonas sphagnophila Christen 1962

count of this species and the values of electrolytic conductivity; Petalomonas sphagnophila occurred in the largest numbers within the range of 40 to $60 \mu \mathrm{S} \mathrm{cm}^{1}$. The research results also revealed the negative (although weak) correlation between the count of the species and the concentration of orthophosphate phosphorus $(r=-0.37)$, nitrogen nitrate $(r=-0.46)$ and ammonia nitrogen $(r=-0.45)$.

It appears from the presented data that this heterotrophic euglenoid preferred acidified habitats with relatively low electrolytic conductivity and low concentrations of main biogenic substances.

From the observations carried out in the reserve Dury, it appears that Petalomonas sphagnophila, as compared with large autotrophic desmids, can represent the r- strategy of development, according to which the species is characterized by immense reproductiveness and a short cycle of biological development (Burchardt 1999). It grows quickly in the environment saturated with light and nutrients, in order to „make it before” other species (Pianka 1981; Kawecka \& Eloranta 1.c.; Reynolds 1988).

\subsubsection{Chroococcus turgidus (Kützing) Nägeli 1849}

Chroococcus turgidus (Fig. 7) - a species from Cyanoprocaryota (Starmach 1966; Matuła 1995; Komárek \& Anagnostidis 1999), morphologically very variable. It occurs in stagnant, also brackish waters, in sludge, between other algae, less frequently in plankton, and in large numbers on wet rocks. It occurs almost permanently on raised bogs among peat mosses. It is a species typical of oligotrophic waters. It is encountered in the littoral zone of stagnant waters, in ponds and lakes. It prefers slightly acidified waters. This cosmopolitan species is encountered both in the soil, 

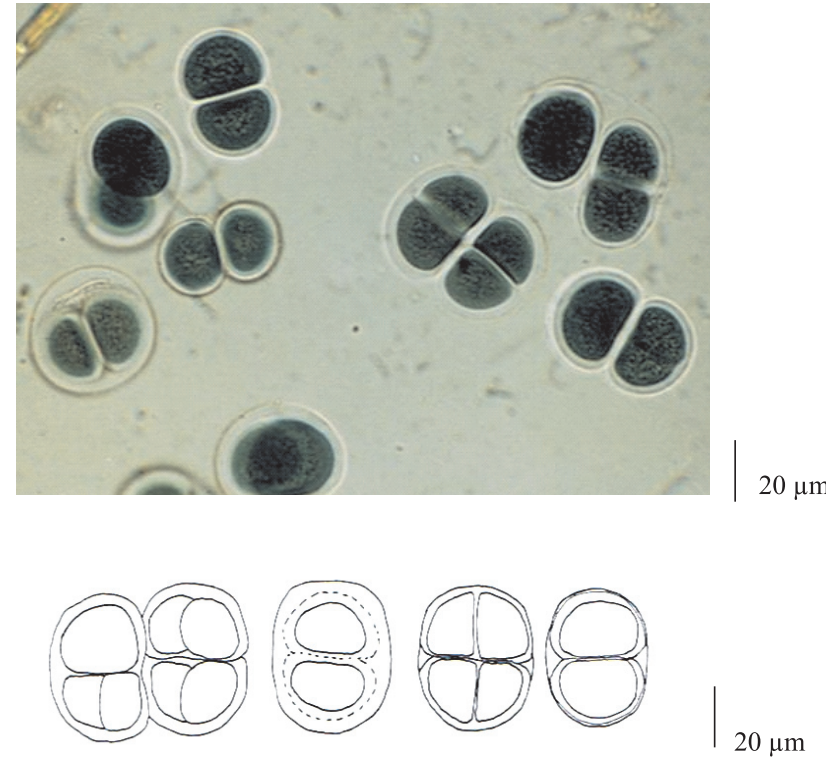

Figure 7. Chroococcus turgidus (Kützing) Nägeli 1849

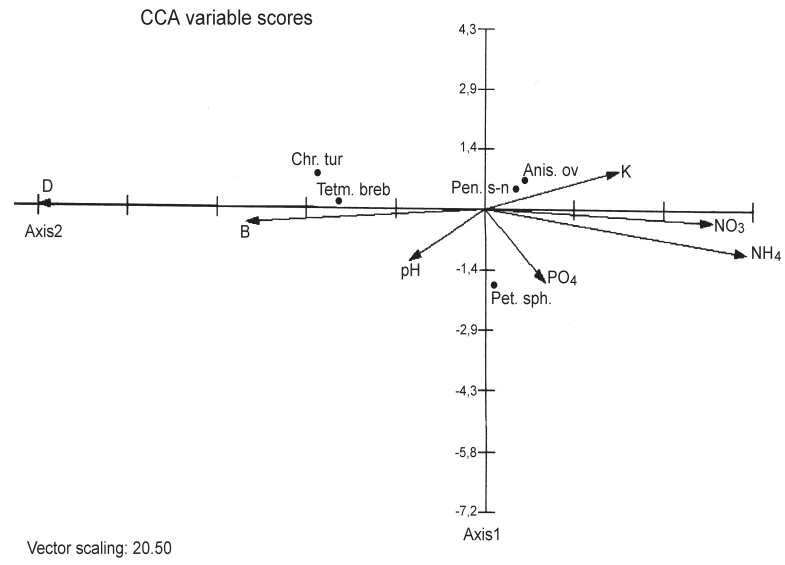

Figure 8. Ordination diagram of the chosen species algae, with axes 1 and 2 of a Canonical Corespondence Analysis (CCA). Applied environmental factors are represented by arrows: $\mathrm{pH}$ - water reaction, $\mathrm{D}$ - distance from water surface, B - Sphagnum biomass of alive to dead ratio, $\mathrm{PO}_{4}, \mathrm{NO}_{3}$ and $\mathrm{NH}_{4}-$ main nutrients concentration in water, $\mathrm{K}$ - conductivity

ents; it is characterized by a wide amplitude of occurrence in relation to $\mathrm{pH}$, however it develops more abundantly in less acidified waters.

This species, likewise Penium silvae-nigrae f. parallelum and Tetmemorus brebissonii, most probably represents the „K development strategy”. It is a large, slowly growing autotroph, presenting the stability in conditions with strong competition of quickly multiplying organisms, dominant during the period of summer stagnation.

\subsection{Influence of physicochemical factors on the distribution of selected algae species}

range of 4.0 and 5.7, reaching the quantitative optimum with $\mathrm{pH}$ 5.0. Undoubtedly the electrolytic conductivity had a strong influence on the growth of cells of Chroococcus turgidus. A strong positive correlation was found between the electrolytic conductivity and the average cell volume, i.e. together with the increased conductivity, the considerable increase of specimens was recorded - from 7,700 to $23,300 \mu \mathrm{m}^{3}$. Also the species count increased from 600 to 430,000 specimens per $\mathrm{dm}^{2}$ of the peat-bog surface area. It appears from the above data that Chroococcus turgidus reached the quantitative and qualitative optimum with low ranges of electrolytic conductivity and low concentration of main biogenic substances in the water.

From the research carried out on the peat bogs Dury, it appears that Chroococcus turgidus is a species typical of highly hydrated habitats, with relatively low values of electrolytic conductivity and small concentrations of nutri-
Relationships between variable habitat conditions and selected algae species are presented with the canonical correspondence analysis (CCA). It appears from the diagram (Fig. 8.) that the distance from the water surface (D) was the major factor differentiating the samples. Another factor influencing the distribution of algae was the electrolytic conductivity of water (C), positively correlated with the 2nd canonical axis. Influence of the remaining parameters was not that distinct. Obviously, also the influence of the water surface and the related humidity on the occurrence of Chroococcus turgidus and Tetmemorus brebissonii was evident. Both species are typical for the littoral zone of dystrophic lakes and as it appears from the performed research - they are stenotopic species associated with a specific habitat, in this case a highly hydrated habitat with low electrolytic conductivity and small concentration of the main bio- 
genic substances. Other species, i.e. Penium silvae-nigrae f. parallelum, Anisonema ovale and Petalomonas sphagnophila did not reveal any affinity to specific habitats. In the studied peat bogs, they occurred in all selected microhabitats, largely tolerating changes in habitat parameters. As it appears from the presented data, the described taxa of algae are - perhaps with the exception of Petalomonas sphagnophila - cosmopolitan (Prescott et al. 1975; Kossinskaja 1960; Starmach 1983; Růžička 1981) and eurytopic organisms.

\section{Summary and conclusions}

In the past, autecology of algae was used mainly to define life requirements and the range of habitat factors for newly discovered and described species (Wojciechowski 2000). At present, autecology deals mainly with studies on relations between organisms and environmental conditions in which they live (Krebs 1997). According to Komárek (2000), ,as a result of the continuous development of further adaptation to new conditions, the existence of genetically and morphologically identical populations in ecologically very different biotopes is quite unlikely". Based on numerous experimental studies, the existence of a large group of algae, with specific ecological requirements and strictly defined ranges of values of environmental parameters, was indeed confirmed. Apart from them, there exist species with broad geographical ranges, capable of adaptation and commonly encountered, but usually only in specifically developed habitats (Komárek 1.c.).

Specific conditions prevailing in the raised bogs make it impossible for many algae species to populate this type of habitats, whereas species that occur here have to demonstrate a high tolerance to both low $\mathrm{pH}$ values and low concentrations of assimilable biogenic substances or periodic shortage of water (Jacuńska 2003a, b). In the community of algae, more autotrophic forms occur at the borderline between the water and the land (Luścińska \& Jakubowska 1999; Jacuńska \& Soska 2002), whereas the number of large autotrophs decreases within the distance close to marshy coniferous forest for the benefit of mixo- and heterotrophic algae. Only desmids remained here, such as Penium silvae-nigrae f. parallelum or Cylindrocystis brebissonii, acidophilous organisms connected with oligotrophic habitats. Also mixotrophic species, such as Anisonema ovale (small, with a rapid reproduction rate) grow in importance here. This phenomenon can be associated with not so much the lack of available nutrients as with water deficiency, at least periodically at sites situated within the outer part of a peat bog.

Species co-dominating at both peat-bogs, such as $\mathrm{An-}$ isonema ovale and Penium silvae-nigrae, despite different nutrition methods and different life „strategies”, have a similarly wide range of tolerance to changeable habitat parameters.

Chroococcus turgidus and Tetmemorus brebissonii are autotrophic species, demonstrating certain characteristics corresponding to the $\mathrm{K}$ - strategy of development. These are species connected with a highly hydrated habitat of low electrolytic conductivity and low concentration of biogenic substances.

\section{References}

Boiński M., 1999, Szata leśna Wdeckiego Parku Krajobrazowego [The forest cover of the Wdecki Landscape Park], Wyd. UMK, Toruń.

Burchardt L., 1999, Ekologia wód, [in:] J. Strzałko and T. Mossor-Pietraszewska (eds.), Kompendium wiedzy o ekologii [Ecology of waters, Compendium of knowledge on ecology], WN PWN, Warszawa-Poznań: 168199.

Choiński A., 1995, Zarys limnologii fizycznej Polski [An outline of physical limnology in Poland], WN UAM, Poznań.

Grime J. P., 1979, Plant strategies and vegetation processes, Wiley, New York.

Hermanowicz W., Dorożańska W., Dojlido I. \& Koziorowski B., 1999, Fizyko-chemiczne badanie wody i ścieków [Physicochemical studies on water and sewage], WN PWN, Warszawa.

Hosiaisluoma V., 1975, Muddy peat algae of Finnish raised bogs, Ann. Bot. Fennici 12: 63-73.

Jacuńska U., 2003a, The algae of transitional and high moor peatbogs in biotopic Gradient, Ecological Questions 3: 93-99.

Jacuńska U., 2003b, The diversity of the species composition and the life strategy of algae in peatbogs surrounding two dystrophic forest lakes, Journal Ecohydrology \& Hydrobiology 4(2): 197-202.

Jacuńska U. \& Soska R., 2002, The influece of variable biotope conditions on the specimen variable and abundance of Penium silvae-nigrae var. Parallelum Krieg. from transitional moor, Ecological Questions 2: 105-109.

Kawecka B. \& Eloranta P. V., 1994, Zarys ekologii glonów wód słodkich i środowisk Lądowych [Introduction to ecology of algae from freshwater and terrestrial environments], WN PWN, Warszawa.

Kępczyński K., 1950, Jeziora Dury w Borach Tucholskich powiat Świecie, Archiwum Wojewódzkiego Konserwatora Przyrody w Bydgoszczy [Lake Dury in Tuchola Forest, the district of Świecie, Archives of the Provincial Nature Conservation Officer in Bydgoszcz], unpublished material.

Kilham P. \& Kilham S.S., 1980, The evolutionary ecology of phytoplankton, [in:] I. Morris (ed.), The physi- 
ological ecology of phytoplankton, Blackwell, Oxford: 571-598.

Komárek I. \& Anagnostidis K., 1999, Cyanoprokaryota, Teil 1, Chroococcales, Gustav Fisher Verlag, StuttgartNew York.

Komárek J., 2000, Ekologia i rozmieszczenie geograficzne cyanoprokariota, Materiały zjazdowe XIX Sympozjum Sekcji Fykologicznej PTB [Ecology and the geographic distribution of cyanoprokaryota, Convention Materials from the 19th Symposium of the Phycological Section of the Polish Botanical Society], Wyd. FIL, Bydgoszcz.

Kossinskaja C. C., 1960, Flora Plantarum Cryptogamarum URSS, Vol. V, Conjugatae (II): Desmidiales, Acad. Scient. URSS, Mosqua-Leningrad.

Kovach W. L., 1993, MVSP - A Multi Variate Statiscal Package for IBM - PC's, ver. 3.1, Kovach Computing Services, Pentraeth, Wales, U.K.

Krebs Ch. J., 1997, Ekologia, Eksperymentalna analiza rozmieszczenia i Liczebności [Ecology, Experimental analysis of the distribution and the count], WN PWN, Warszawa.

Lampert W. \& Sommer U., 1996, Ekologia wód śódlądowych [Ecology of inlan dwaters], WN PWN, Warszawa.

Law R., Bradshaw A. D. \& Putwain P. D., 1977, Life history variation in Poa annua, Evolution 31: 46-233.

Luścińska M. \& Jakubowska U., 1999, Dynamics of abundance and development of algae in humic lake in Tuchola Forest, Acta Universitatis Nicolai Copernici, Limnological Papers 20, Nauki Mat.-Przyr. 101: 69-84.

MacArthur R. H., 1962, Some generalized theorems of natural selection, Proc. Nat. Acad. Sci. 48: 1893-1897.

MacNaughton S. J., 1975, r- and k-selection in Typha, American Naturalist 109: 251-261.

Margalef R., 1978, Life-forms of phytoplankton as survival alternatives in an unstable Environment, Oceanol. Acta 1: 493-509.

Matuła J., 1995, Warunki troficzne glonów torfowiskowych na obszarze Dolnego Śląska [Trophic conditions of peat-bog algae in Lower Silesia], Katedra Botaniki i Fizjologii Roślin, Uniwersytet Wrocławski [Department of Botany and Plant Physiology, University of Wrocław], Wrocław.

Nienartowicz A., Boińska U. \& Boiński M., 1988, Zastosowanie metod numerycznych do analizy przestrzennego zróżnicowania roślinności na terenie rezerwatu Dury w Borach Tucholskich [Application of numerical methods in the analysis of spatial heterogeneity of vegetation in the reserve of Dury in the Tuchola Forest], Acta Universitatis Nicolai Copernici, Biologia 32, Nauki Mat.-Przyr. 69: 225-236.

Oleksowicz A. S., 1987, Uwagi o przydatności desmidii (Chlorophyta, Conjugatophyceae) jako wskaźnika czystości i żyzności wód na tle czynników kontrolujących ich występowanie [Remarks on the usefulness of desmids (Chlorophyta, Conjugatophyceae) as an index of water purity and fertility with reference to factors controlling their occurrence], Wiad. Bot. 31(2): 73-85.

Palmer R. W., 1998, Canonical Correspondence Analysis: some advice and guidelines, [in:] E. Kaźmierczak, A. Nienartowicz, A. Piernik, J. Wilkoń-Michalska (eds.), Metody numeryczne w badaniach struktury i funkcjonowania szaty roślinnej [Numerical methods in the studies on the structure and functioning of the vegetation cover], Wyd. UMK, Toruń: 171-187.

Pianka E. R., 1981, Ekologia ewolucyjna [Evolutionary ecology], PWN, Warszawa.

Prescot G. W., Croasdale H. T. \& Vinyard W. C., 1975, A symposis of North American desmids, Part II, Desmidiaceae: Placodermae, Section 1, University of Nebraska Press, Lincoln.

Růžička J., 1981, Die Desmidiaceen Mitteleuropas, Bd. 1, E. Schweizerbert'sche Verlagsbuchhandlung, Stuttgart.

Reynolds C. S., 1984, The Ecology of Freshwater Phytoplankton, Cambridge University Press, Cambridge.

Reynolds C. S., 1988, Functional morphology and the adaptive strategies of freshwater phytoplankton, Cambridge University Press, Cambridge: 388-433.

Reynolds C. S., 1993, Scales of disturbance and their role in plankton ecology, Hydrobiologia 249: 157-171.

Reynolds C. S., 1995, Succssional change in the planktonic vegetation: species, structures, scales, [in:] I. Joint (ed.), Molecular Ecology of Aquatic Microbes, Springer-Verlag, Berlin: 115-32.

Reynolds C. S., 2006, The Ecology of Phytoplankton, Cambridge University Press, Cambridge.

Smayda T. J. \& Reynolds C. S., 2003, Strategies of marine dinoflagellate survival and some rules of assembly, Journal of Sea Research 49: 95-106.

Sommer U., 1981, The role of $r$ - and $K$-selection in the succession of phytoplankton in Lake Constance, Acta Oecologia 2: 327-342.

Sommer U., 1988, Growth and survival strategies of planctonic diatoms, Cambridge University Press, Cambridge: $227-260$.

Starmach K., 1966, Cyanophyta - Sinice, Glaucophyta - Glaukofity, Flora słodkowodna Polski [Cyanophyta - Blue-green algae, Glaucophyta, Freshwater flora of Poland], WN PWN, Warszawa.

Starmach K., 1983, Euglenophyta - Eugleniny, Flora słodkowodna Polski T. 3 [Euglenophyta - Euglenoids. Freshwater flora of Poland Vol. 3], WN PWN, Warszawa.

Turpin D. H., 1988, Physiological mechanisms in phytoplankton resource Competition, Cambridge University Press, Cambridge: 316-368. 
Van Der Maarel E., 1998, Multivariate analysis in plant ecology [in:] E. Kaźmierczak, A. Nienartowicz, A. Piernik and J. Wilkoń-Michalska (eds), Metody numeryczne w badaniach struktury i funkcjonowania szaty roślinnej [Numerical methods in the studies on the structure and functioning of the vegetation cover], Wyd. UMK, Toruń: 65-108.

Walas J., 1950, Rezerwat wodny i torfowiskowy Dury w Nadleśnictwie Osie, Archiwum Wojewódzkiego Konserwatora Przyrody w Bydgoszczy [The water and peat bog nature reserve of Dury in the Forest District of Osie. Archives of the Provincial Nature Conservation Officer in Bydgoszcz], (unpublished material).

Weithoff G., Lorke A. \& Walz N., 2000, Effects of watercolumn mixing on bacteria, phytoplankton and rotifers under different levels of herbivory in a shallow eutrophic lake, Oecologia 125: 91-100.

Wojciechowski I., 1987, Ekologiczne podstawy kształtowania środowiska [Ecological basis of environmental architecture], WN PWN, Warszawa.
Wojciechowski I., 1999, Warunki funkcjonowania ekosystemów torfowiskowych i wodno - torfowiskowych w Polsce, Materiały Międzynarodowej Konferencji Problemy aktywnej ochrony ekosystemów wodnych i torfowiskowych w polskich parkach narodowych, [Functioning conditions of peat-bog and water - peatbog ecosystems in Poland, Materials of the International Conference - Problems with active protection of aquatic and peat-bog ecosystems in Polish national parks], Wydawnictwo UMCS, Lublin: 57-64.

Wojciechowski I., 2000, Ekologia glonów jako współmałżonek taksonomii, Materiały izjazdowe XIX Sympozjum Sekcji Fykologicznej PTB, [Ecology of algae as a spouse of taxonomy, Convention Materials from the 19th Symposium of the Phycological Section of the Polish Botanical Society], Wydawnictwo FIL, Bydgoszcz.

Wołowski K., 1998, Taxonomic and environmental studies on euglenophytes of the Kraków - Częstochowa upland (Southern Poland), Fragm. Flor. Geobot. Supplementum 6, Kraków. 